\title{
Expression of combined interference of slug and FoxC2 in endometrial carcinoma and its clinicopathological relationship
}

\author{
Qing $\mathrm{Zhu}^{1,2 *}$, Mingyang Tang ${ }^{3}$, Ligao $\mathrm{Wu}^{1,2 * \wedge}$ \\ ${ }^{1}$ Departments of Pathology, The First Affiliated Hospital of Bengbu Medical College, Bengbu, China; ${ }^{2}$ Department of Pathology, Bengbu Medical \\ College, Bengbu, China; ${ }^{3}$ Clinical Medicine Science, School of Clinical Medicine, Bengbu Medical College, Bengbu, China \\ Contributions: (I) Conception and design: L Wu; (II) Administrative support: L Wu; (III) Provision of study materials or patients: M Tang; (IV) \\ Collection and assembly of data: M Tang, L Wu; (V) Data analysis and interpretation: Q Zhu, M Tang; (VI) Manuscript writing: All authors; (VII) \\ Final approval of manuscript: All authors. \\ "These authors contributed equally to this work. \\ Correspondence to: Ligao Wu. Departments of Pathology, The First Affiliated Hospital of Bengbu Medical College, 287 Changhuai Road, Longzihu \\ District, Bengbu 233000, China; Departments of Pathology, Bengbu Medical College, No.2600 Donghai Avenue, Longzihu District, Bengbu \\ 233030, China. Email: ahwuligao@126.com.
}

Background: Endometrial carcinoma is one of the three major malignant tumors in female reproductive system. The current research aimed to investigate the relationship between the Slug and FoxC2 expression and the proliferation, invasion and metastasis of endometrial carcinoma.

Methods: The expression of Slug and FoxC2 genes between 124 endometrial carcinoma tissues and 35 normal endometrial tissues was analyzed through immunohistochemistry. The endometrial carcinoma cell lines Ishikawa and RL-952 were cultured, the Slug-shRNA and FoxC2-shRNA expression vectors were constructed, and the endometrial carcinoma cells interfering with the expression of Slug and FoxC2 genes were also established. Western blotting and RT-PCR were employed to verify whether shRNA could down regulate the expression of Slug and FoxC2 genes. Additionally, the proliferation, migration and invasion capacities in both cell lines after interfering with Slug and FoxC2 was detected through CCK-8 and Transwell assay respectively. Furthermore, MMP2 and MMP9 were detected by ELISA and epithelialmesenchymal transition (EMT) related proteins including E-cadherin, $\mathrm{N}$-cadherin and Vimentin were assessed by Western blotting analysis.

Results: Compared with normal endometrial tissues, the Slug and FoxC2 expression levels in endometrial carcinoma tissues were remarkably increased. shRNAs transfection significantly down-regulated expressions in both endometrial carcinoma cell lines. The proliferation, invasion and migration ability were significantly inhibited by Slug-shRNA and FoxC2-shRNA compared with the control group. The expression of E-cadherin was increased while the expression of N-cadherin, Vimentin, MMP-2 and MMP-9 was suppressed by the Slug-shRNA and FoxC2-shRNA.

Conclusions: Slug and FoxC2 could be used as a prognostic factor of endometrial carcinoma. Interfering with the expression of Slug and FoxC2 in endometrial carcinoma cell lines could effectively inhibit the proliferation, invasion and migration, and its mechanism is related to the inhibition of EMT. Slug and FoxC2 are potential targets for the treatment of endometrial carcinoma.

Keywords: Endometrial carcinoma; gene targeting regulation; Slug; FoxC2; epithelial-mesenchymal transition (EMT)

Submitted Feb 04, 2020. Accepted for publication Jul 03, 2020.

doi: $10.21037 /$ tcr-20-809

View this article at: http://dx.doi.org/10.21037/tcr-20-809

^ ORCID: 0000-0001-5819-8847. 


\section{Introduction}

Endometrial carcinoma is one of the most malignant tumors in female reproductive system, which has severely damaged the life health in women. However, there is still lacking effective prognostic indicators and treatment options for the clinical treatments. Slug is a transcription factor of SNAIL family that can encode the zinc finger protein, which is reported to be up-regulated in multiple malignant tumors. FoxC2 is a member of the Fox protein family, which can regulate the expression of a series of downstream genes, and participate in the epithelial-mesenchymal transition (EMT) (1-3). Transcription factors Slug and FoxC2 have been reported to play important roles in tumor invasion and metastasis, which are the important regulatory molecules during tumor cell migration and metastasis (4).

Short hairpin RNA (shRNA) is a kind of RNA sequence that can form a tight hairpin turn, which can silence gene expression. The double-strand small molecule RNA or shRNA has been utilized in clinical test to treat several diseases, and certain achievements have been attained in terms of tumor treatment. In the current study, shRNAs were used to down-regulate the Slug and FoxC2 expression in Ishikawa and RL-952 endometrial carcinoma cell lines, and cell proliferation, migration and invasion were further evaluated. Then we tested the relevant indicators of EMT after transfection to gain a deeper understanding of the role of Slug and FoxC2 in endometrial carcinoma.

We present the following article in accordance with the MDAR checklist (available at http://dx.doi.org/10.21037/ tcr-20-809).

\section{Methods}

\section{Patients}

The endometrial carcinoma tissues were retrospectively collected from 124 patients in the Pathology Department of the First Affiliated Hospital of Bengbu Medical College from January 2014 to December 2015. Before surgery, all endometrial carcinoma patients did not receive any anticancer treatments, including chemotherapy and radiotherapy. Patients with endometrial carcinoma were confirmed by pathologic diagnosis. The normal endometrial tissues were collected into the control group, which were derived from 35 patients receiving endometrial tissue biopsy and hysterectomy due to other benign disease in Gynecology.

The study was conducted in accordance with the
Declaration of Helsinki (as revised in 2013). The study was approved by institutional ethics board of The First Affiliated Hospital of Bengbu Medical College (No. BBMEC-2018-10) and informed consent was taken from all the patients.

\section{Immunobistochemical staining}

Endometrial tissues resected from uterus of patients, were rinsed with normal saline, fixed with $4 \%$ formalin for $24 \mathrm{~h}$, dehydrated and embedded. The samples were sliced into sections $0.4 \mu \mathrm{m}$ in thickness, followed by dewaxing of the sections and antigen retrieval in citric acid solution for $3 \mathrm{~min}$ at $121^{\circ} \mathrm{C}$. After natural cooling, the sections were treated with $3 \% \mathrm{H}_{2} \mathrm{O}_{2}$ solution for $10 \mathrm{~min}$, followed by the addition of anti-Slug (ab180714, Abcam, Cambridge, MA, USA) and anti-FoxC2 (ab5060, Abcam, Cambridge, MA, USA) rabbit anti-human primary antibodies to incubate for $1 \mathrm{~h}$ at $60{ }^{\circ} \mathrm{C}$, rinsing with PBS for $3 \times 5$ min and incubation with secondary antibody for $30 \mathrm{~min}$ at $37^{\circ} \mathrm{C}$. Then, the sections were washed with PBS, followed by DAB staining for visualization, nuclear counterstaining of hematoxylin and microscopic observation. The Image-Pro Plus software was adopted for image analysis by another person who was blind to the group assignments, and the integrated optical density/area ratio was used as the criterion to determine the protein expression contents of Slug and FoxC2.

\section{Cell culture and transfection}

Human endometrial carcinoma cell lines Ishikawa (catalog number BH-X5152, Shanghai Bohu Biotechnology Co., Ltd.) and RL-952 (catalog number BNN1364, Shanghai Beinuo Biology Co., Ltd.) were maintained in RPMI 1640 medium (HyClone, Thermo Scientific) supplemented with $10 \%$ fetal bovine serum, $100 \mathrm{U} / \mathrm{mL}$ penicillin and $100 \mathrm{U} / \mathrm{mL}$ streptomycin, and incubated in a humidified incubator under the conditions of $5 \% \mathrm{CO}_{2}$ and $95 \%$ air at $37{ }^{\circ} \mathrm{C}$. Subsequently, Ishikawa and RL-952 cells at logarithmic growth phase were collected and seeded randomly into a 96well plate $\left(2 \times 10^{4}\right.$ cells $\left./ \mathrm{mL}\right)$ and cultured for $24 \mathrm{~h}$. The $2-5$ generations cells were used for the following experiments.

The target plasmid DNA and Lipofectamine 2000 were diluted in the serum-free Opti-MEM. Cells at the density of $60-70 \%$ were collected and incubated with the mixed medium for $20 \mathrm{~min}$ at room temperature. Then, the mixtures were added to the 96-well plate containing both cells and culture solution, agitated gently, and placed in the 
incubator. The Slug-shRNA and FoxC2-shRNA expression vector (GenePharma, Shanghai, China) treatment groups (containing cells, complete medium, Slug-shRNA and FoxC2-shRNA), negative control group (containing cells, complete medium, and negative control-shRNA), and blank control group (containing cells and complete medium) were set, and 4 duplicates were prepared for each group.

\section{Protein expression changes detected by Western blotting}

Cells were harvested at $48 \mathrm{~h}$ after transfection, and lysed with the cell lysis buffer. Equivalent amounts of the sample lysate were then separated through sodium dodecyl sulfate polyacrylamide gel electrophoresis, which were later transferred onto the nitrocellulose membrane (Millipore) by electroblotting. Later, the membrane was blocked with $5 \%$ non-fat milk in the TBST buffer (containing $20 \mathrm{mM}$ Tris$\mathrm{HCl}, \mathrm{pH} 7.4,150 \mathrm{mM} \mathrm{NaCl}$ and $0.1 \%$ Tween 20 ) at $4{ }^{\circ} \mathrm{C}$ overnight, followed by incubation with specific primary antibodies for $2 \mathrm{~h}$ and the specific IgG HRP-conjugated secondary antibody for $1 \mathrm{~h}$ at room temperature. Details for the primary antibodies were as follows: Slug (1:2,000, catalog number \# ab180714) and FoxC2 (1:2,000, catalog number \#ab5060) were obtained from Abcam (Cambridge, MA, USA), $\beta$-actin (1:1,000, catalog number \#bs-10021) was purchased from Bioworld (CA, USA). The resultant signals would then be visualized through enhanced chemiluminescence (ECL) (Pierce) on the Syngene G: BOX Chemi gel documentation system (Syngene, Cambridge, UK). The densitometric values would be normalized in each group, with $\beta$-actin being used as the internal reference.

\section{Real-time quantitative PCR analysis}

Cells in each group were harvested at $48 \mathrm{~h}$ after transfection, and the total RNA would then be isolated using the TRIzol kit (Invitrogen, CA, USA) in accordance with the manufacturer's instructions. $1 \mu \mathrm{g}$ total RNA was used to synthesize cDNA using a PrimeScript reagent Kit with DNAEraser (Takara Bio, Kyoto, Japan), followed by RT-PCR analysis using the SYBR green Master Mix (Takara Bio, Kyoto, Japan). The following primer pairs were obtained from Invitrogen (NY, USA), while GAPDH served as the internal reference.

GAPDH: 5'-GAAGAGTCAAGAGCAGTCGTCAAG A-3' (forward), 5'-GTAAGACCGAACCACAGTCAAGAG AG-3' (reverse); Slug: 5'-GAGCCTTCAAGAGAAAAAAG GAATT-3' (forward), 5'-GTAGTCAAGAGTCTTTTTT
CCTTAA-3' (reverse); FoxC2: 5'-GAGCCTTCAAGAGC CAGGTGGAATT-3' (forward), 5'-GTTCCGTCTTCTC AAGAGACGAATA-3' (reverse).

\section{CCK-8 colorimetry assay}

Ten $\mu \mathrm{L}$ CCK- 8 was added into each well at 24, 48, 72 and $96 \mathrm{~h}$, respectively, and further cultured for $4 \mathrm{~h}$, and the optical density value in each well was measured using a microplate reader. Subsequently, the growth curve was plotted, and the average of 4 wells in each group was used to calculate the cell proliferation capacity. Each experiment was repeated for three times.

\section{Transwell chamber migration assay}

Cells in each group were digested with trypsin and washed with the serum-free culture medium. Cell suspension was prepared, and $200 \mu \mathrm{L}$ was evenly added into the upper Transwell chamber (Beyotime Biotechnology, Haimen, China), while $600 \mu \mathrm{L}$ complete medium was added to the lower Transwell chamber for $48 \mathrm{~h}$. Then, the chambers were taken out, washed with PBS, fixed with $4 \%$ paraformaldehyde for $20 \mathrm{~min}$, and stained with $1 \%$ crystal violet staining solution for $10 \mathrm{~min}$. Subsequently, cells on the basilar membrane surface were wiped using the cotton swab, and the number of cells penetrating the membrane was counted under microscope. Each experiment was repeated for three times.

\section{Transwell invasion assay}

Before Transwell invasion assay, cells were deprived of serum for $24 \mathrm{~h}$, resuspended $\left(5 \times 10^{4}\right)$ with $200 \mu \mathrm{L}$ serumfree medium after trypsin digestion, and added onto the Matrigel surface in the upper Transwell chamber, while $600 \mu \mathrm{L}$ complete medium was added to the lower Transwell chamber as the chemotaxin. The number of cells penetrating the Matrigel was counted under microscope. Each experiment was repeated for three times.

\section{ELISA assay}

The concentrations of MMP-2 and MMP-9 were further evaluated in both cell lines. Cell culture supernatants were collected at $48 \mathrm{~h}$ after transfection. MMP-2 and MMP9 concentrations were measured using human MMP2 ELISA Kit and human MMP-9 ELISA Kit (Invitrogen 

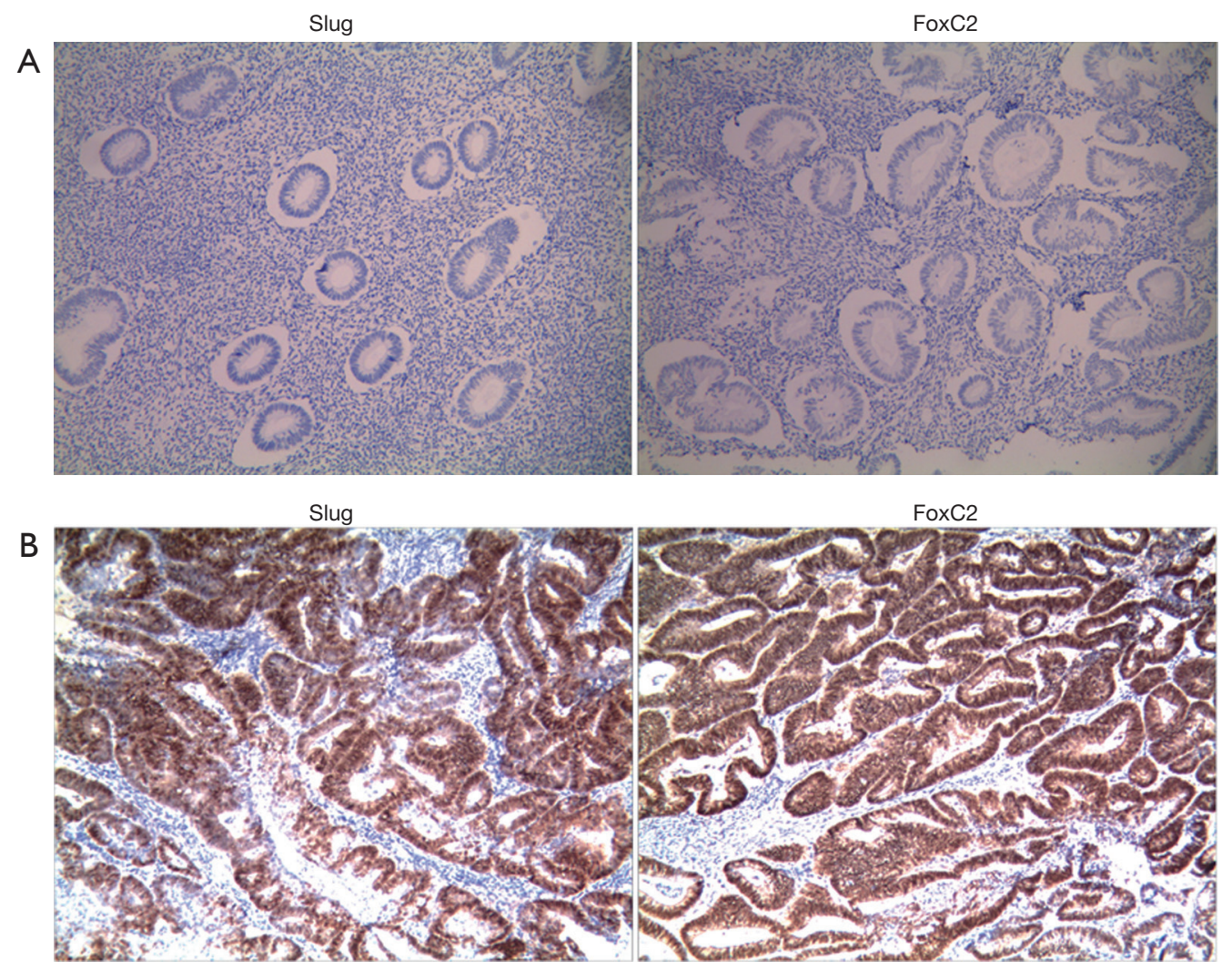

Figure 1 Expression of Slug and FoxC2 in the normal control (A) and endometrial carcinoma (B) tissues was analyzed using immunohistochemical staining $(\times 100)$.

Corporation, Carlsbad, United States) according to the manufacturer's instructions. The optical density (OD) value at $450 \mathrm{~nm}$ was record using a microplate reader.

\section{Statistical analysis}

Statistical analyses were performed using SPSS 18 for Windows (SPSS Inc., Chicago, IL, USA). Data are given as means \pm SD and One-way analysis of variance followed by post-hoc Bonferroni analysis of variance. The two groups were compared with Student's t-test. Each experiment was repeated for three times. $\mathrm{P}<0.05$ was taken as a significant difference between groups.

\section{Results}

\section{Slug and FoxC2 were bighly expressed in endometrial carcinoma tissues}

As shown in Figure 1, immunohistochemical results indicated positive expression in the nuclei of endometrial carcinoma tissues. Compared with normal endometrial tissues, the Slug and FoxC2 expression levels in endometrial carcinoma tissues were remarkably increased. The positive rate of Slug and FoxC2 in endometrial carcinoma was $61.3 \%(76 / 124)$ and $71.0 \%(88 / 124)$, and the difference was statistically significant compared with that in normal endometrial tissues (Table 1).

\section{Relationships of Slug and FoxC2 expression with the clinicopathological factors of endometrial carcinoma}

There was no relationship between the Slug and FoxC2 expression and age in each group $(\mathrm{P}>0.05)$. Slug expression in endometrial carcinoma was notably correlated with the histological grade, muscular layer infiltration and lymph node metastasis $(\mathrm{P}<0.05)$, but it was not markedly correlated with The International Federation of Gynecology and Obstetrics (FIGO) stage. FoxC2 expression was distinctly related to the FIGO stage and lymph node metastasis $(\mathrm{P}<0.05)$, but it was not correlated with histological grade 
Table 1 Expression of Slug, FoxC2 in distinct tissue types

\begin{tabular}{|c|c|c|c|c|c|c|c|}
\hline Histological type & Cases & \multicolumn{3}{|c|}{ Slug } & \multicolumn{3}{|c|}{ Fox } \\
\hline Normal endometrial tissues & 35 & 27 & 8 & $<0.01$ & 31 & 4 & $<0.01$ \\
\hline Endometrial carcinoma tissues & 124 & 48 & 76 & & 36 & 88 & \\
\hline
\end{tabular}

$\mathrm{P}<0.01$ vs. normal and atypical hyperplastic group.

Table 2 The association of Slug, FoxC2 expression with clinicopathological features in endometrial carcinoma

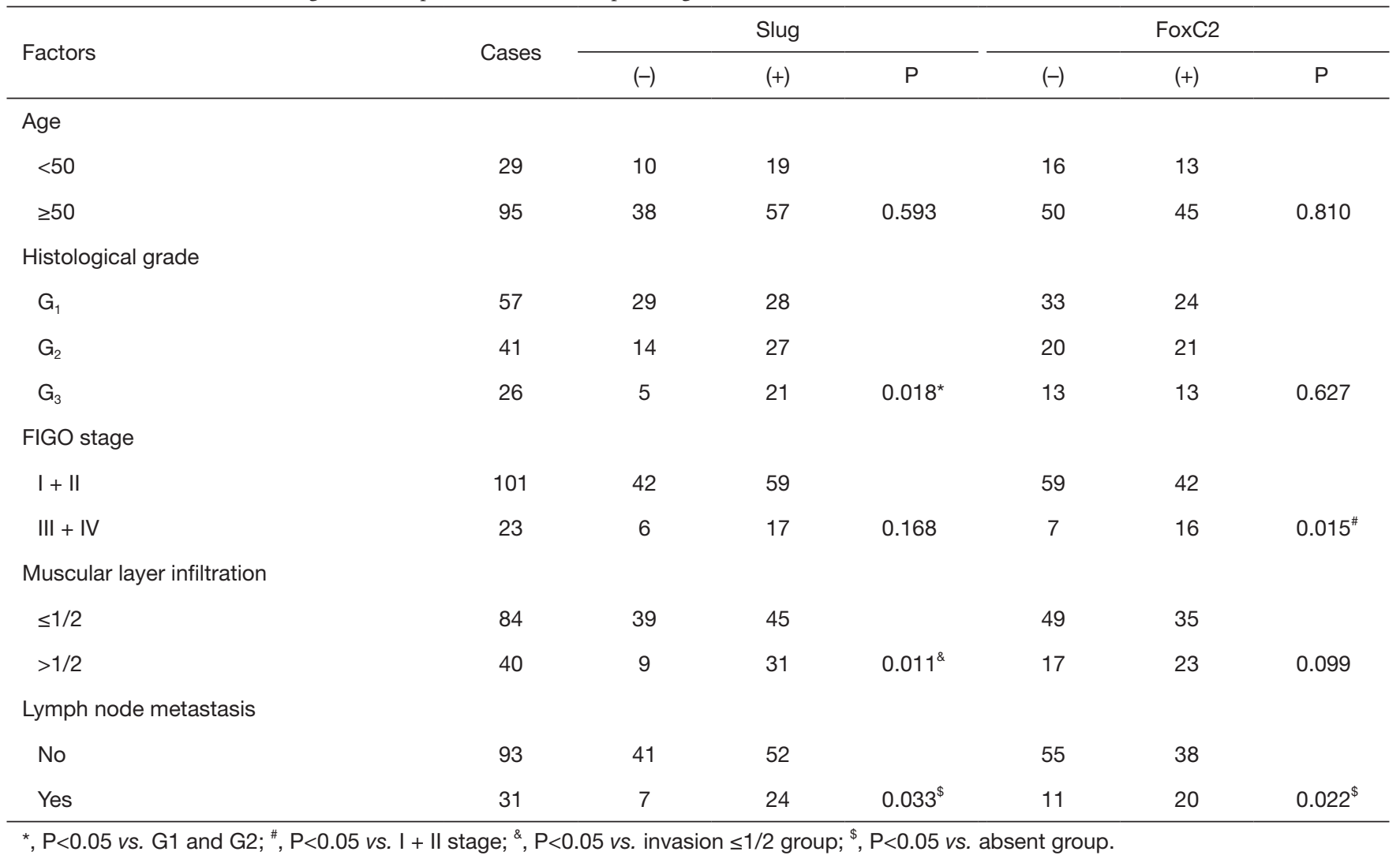

and muscular layer infiltration (Table 2).

\section{Slug and FoxC2 protein expression were detected by Western blotting and RT-PCR}

Ishikawa and RL-952 cells were first observed at $48 \mathrm{~h}$ after shRNAs transfection (Figure 2). The fluorescence images indicated that the cells were successfully transfected with the shRNAs. Western blotting and RT-PCR results also indicated that, compared with control group, Slug and FoxC2 protein and mRNA levels in Ishikawa (Figure 3) and RL-952 (Figure 4) cells were remarkably reduced after Slug-
shRNA and FoxC2-shRNA interference $(\mathrm{P}<0.01)$. Nonspecific shRNA group showed no significant difference compared with those in blank control group $(\mathrm{P}>0.05)$.

\section{Slug-shRNA and FoxC2-shRNA inbibited endometrial carcinoma cell proliferation}

As shown in Figure $3 D$ and Figure $4 D$, the proliferation rates of both cell lines in the control group were markedly higher than those in shRNAs treatment groups. Independent sample $t$-test results suggested that, the differences in the proliferation capacity of cells in expression vector treatment 


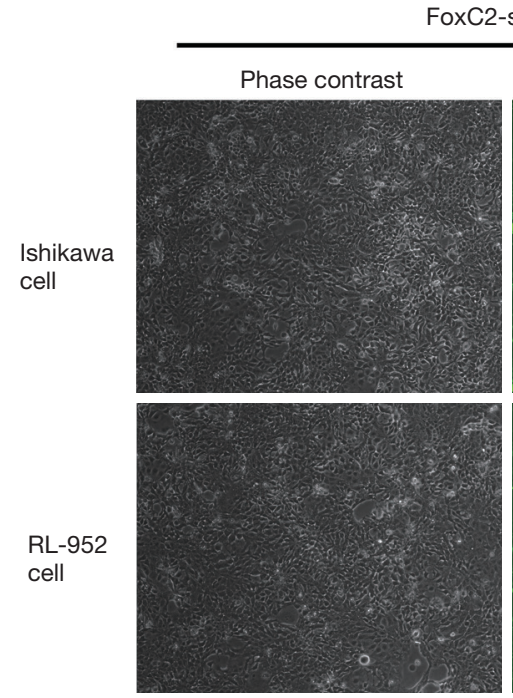

oxC2-shRNA
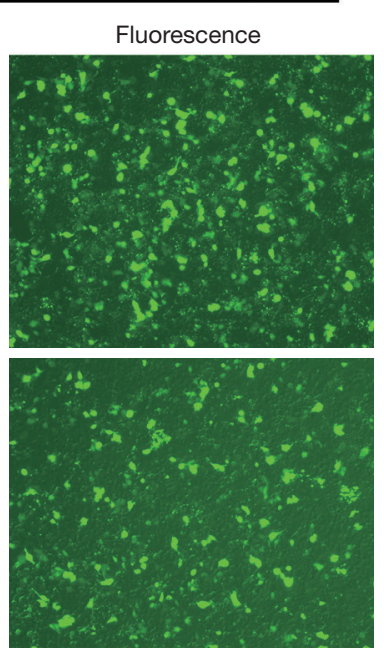

Slug-shRNA

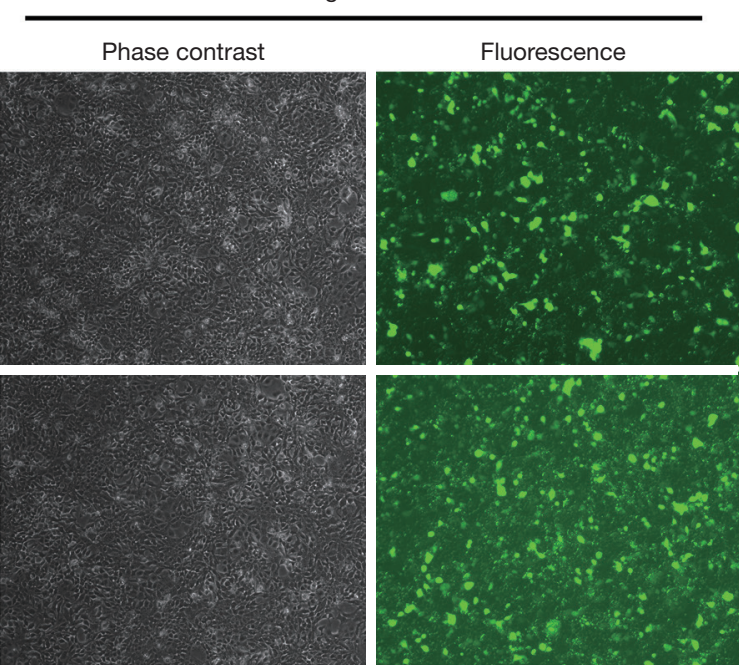

Figure 2 Ishikawa and RL-952 cells were observed at 48 h after transfection with Slug-shRNA and FoxC2-shRNA ( $\times 100)$.

groups were statistically significant compared with that in the other two groups at days 2, 3, 4, 5, 6 and 7 of growth $(\mathrm{P}<0.05)$. CCK- 8 assay suggested that, the proliferation capacity of cells in shRNAs treatment groups was apparently lower than that in the control group.

\section{Slug-shRNA and FoxC2-shRNA inbibited endometrial carcinoma cell migration and invasion}

The migration and invasion abilities in Ishikawa and RL952 cells were then evaluated. The numbers of Ishikawa cells penetrating the filter membrane into the lower chamber in Slug-shRNA and FoxC2-shRNA groups were $174.58 \pm 8.11$ and $165.33 \pm 7.65$, respectively. The numbers of cells penetrating Matrigel into the lower chamber in SlugshRNA and FoxC2-shRNA groups were $94.02 \pm 6.57$ and $84.56 \pm 8.36$, respectively. While those in the control group and blank control group were $261.76 \pm 10.11$ in migration assay and $175.02 \pm 9.65$ in invasion assay (Figure 5). The results in RL-952 cell lines were similar with the Ishikawa cells. The numbers of cells penetrating the filter membrane into the lower chamber in Slug-shRNA and FoxC2-shRNA groups were $148.72 \pm 6.54$ and $136.48 \pm 5.22$, respectively. The numbers of cells penetrating Matrigel into the lower chamber in Slug-shRNA and FoxC2-shRNA groups were $76.42 \pm 5.63$ and $77.82 \pm 7.32$, respectively. While those in the control group $198.52 \pm 9.72$ in migration assay and $178.63 \pm 6.38$ in invasion assay (Figure 6). Compared with the control group, the numbers of cells entering the lower chamber in Slug-shRNA and FoxC2-shRNA groups in both cell lines were significantly reduced $(\mathrm{P}<0.05)$.

\section{Slug-shRNA and FoxC2-shRNA reduced MMP-2 and MMP-9 in endometrial carcinoma cells}

Subsequently, we compared the MMP-2 and MMP-9 of the cell culture supernatant in each group of both cell lines by ELISA assay. As shown in Figure 7, the expressions of MMP-2 and MMP-9 after shRNA-Slug and shRNA-FoxC2 transfection were significantly reduced. This is consistent with the results of our previous migration and invasion results.

\section{shRNA-Slug and shRNA-FoxC2 inbibited EMT in endometrial carcinoma}

To gain insights into the mechanism underlying the Slug and FoxC2 inhibiting endometrial carcinoma migration and invasion, we evaluated the protein level of EMT related protein including E-cadherin, N-cadherin and Vimentin by Western blotting. As shown in Figure 8, after transfection of shRNA-Slug and shRNA-FoxC2 in Ishiyama and RL-952 endometrial carcinoma cells, the expression of E-cadherin increased while the expression of $\mathrm{N}$-cadherin and Vimentin decreased. These results indicated that EMT capability is suppressed by shRNA-Slug and shRNA-FoxC2. 
A
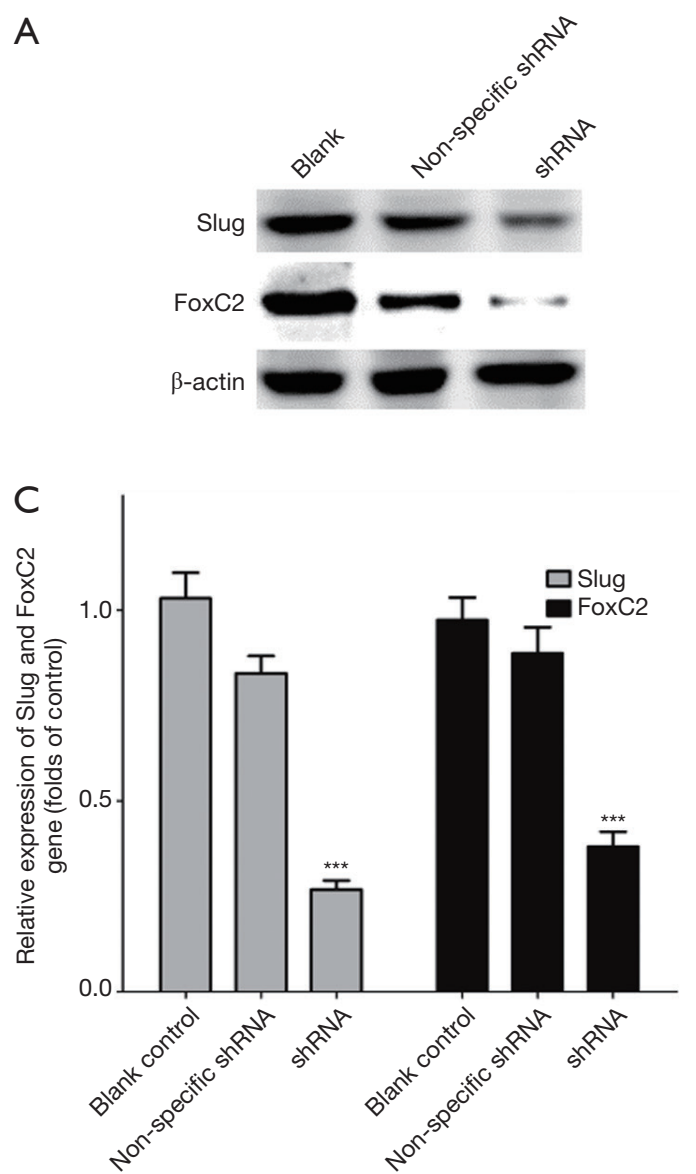

B
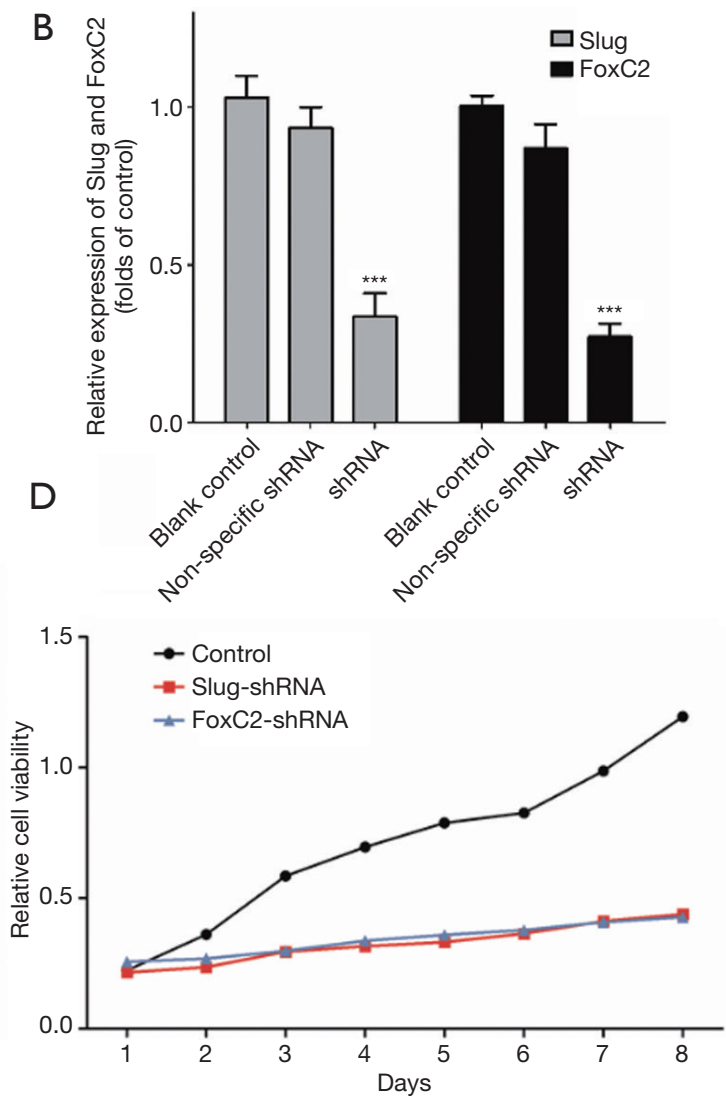

Figure 3 Slug-shRNA and FoxC2-shRNA reduced Ishikawa cells proliferation. (A) The protein expression in each group was detected using western-blot. Representative images for Slug and FoxC2 were shown; (B) quantification of Slug and FoxC2 expression; (C) the Slug and FoxC2 gene in each group was detected by RT-PCR; (D) Ishikawa cell viability was detected on the on the 1-8 days after transfection using CCK-8 method. Data represent the mean $\pm \mathrm{SD}(\mathrm{n}=3){ }^{* * *}, \mathrm{P}<0.001$ versus control group.

\section{Discussion}

In the current study, we found that Slug and FoxC2 expressions in endometrial carcinoma tissues were remarkably increased. In addition, Slug and FoxC2 silences by shRNA significantly inhibited endometrial carcinoma proliferation and metastasis, which may be related to the downregulation of MMPs protein expression and inhibition of EMT.

As a member of the Zinc finger transcription factor family members, Slug is mainly involved in neoplasm malignant phenotype regulation, and could also promote EMT, which is closely related to tumor cell migration and invasion $(2,5)$. Recent researches have indicated that, Slug antisense could prevent EMT, indicating that, the Slug gene could act a treatment target for tumor invasion and metastasis (6-8).

FoxC2 is also referred to as mesenchyme forkhead 1 (MFH1), which belongs to the forkhead transcription factor family member and is encoded by genes located at human chromosome 16q24.1 $(9,10)$. It has been highlighted that FoxC2 plays a central role in multiple signaling pathways, which are closely related to EMT and could further promote angiogenesis in tumor development $(11,12)$.

In our current study, we found that the positive expression rates of Slug and FoxC2 proteins in endometrial carcinoma of $68.75 \%$ and $59.34 \%$, respectively, which were remarkably elevated compared with that in normal endometrial tissue group (25.00\%). Besides, the Slug and FoxC2 expressions were evidently correlated with the endometrial carcinoma development and poor prognosis 
A
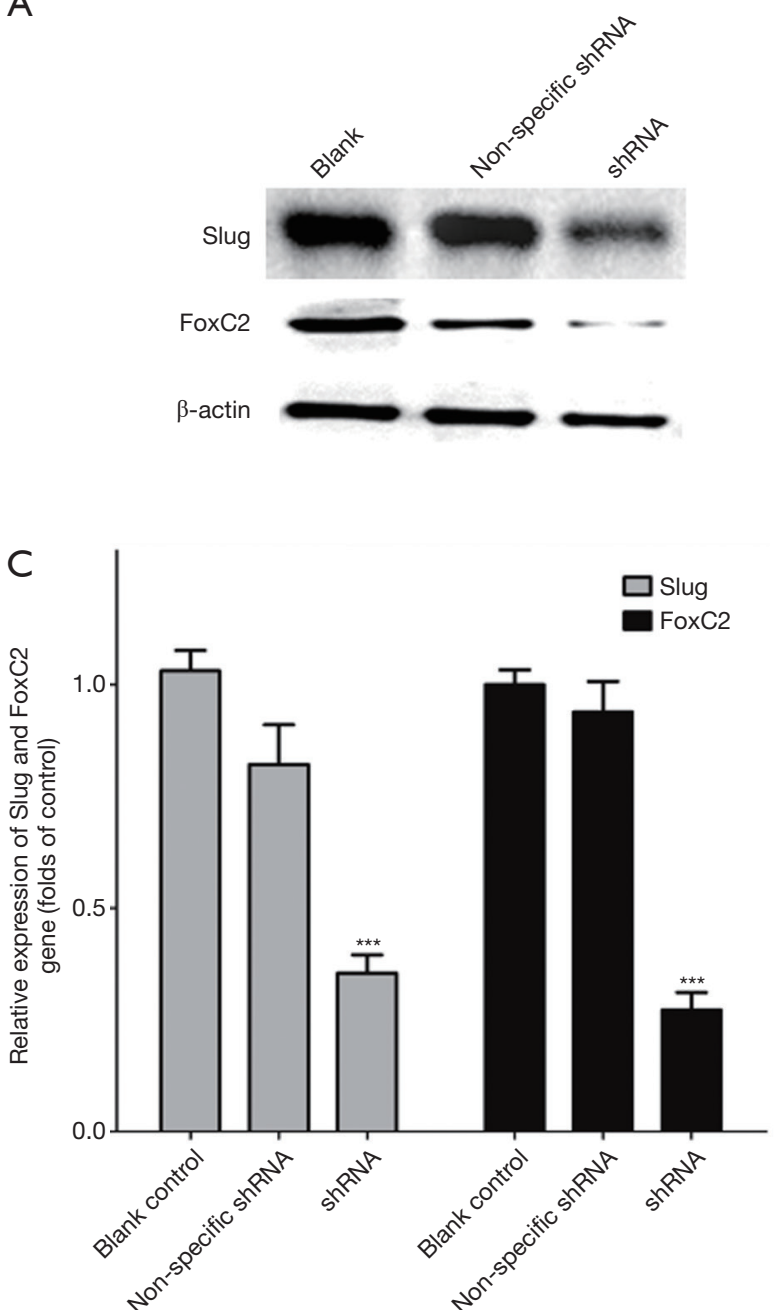

B
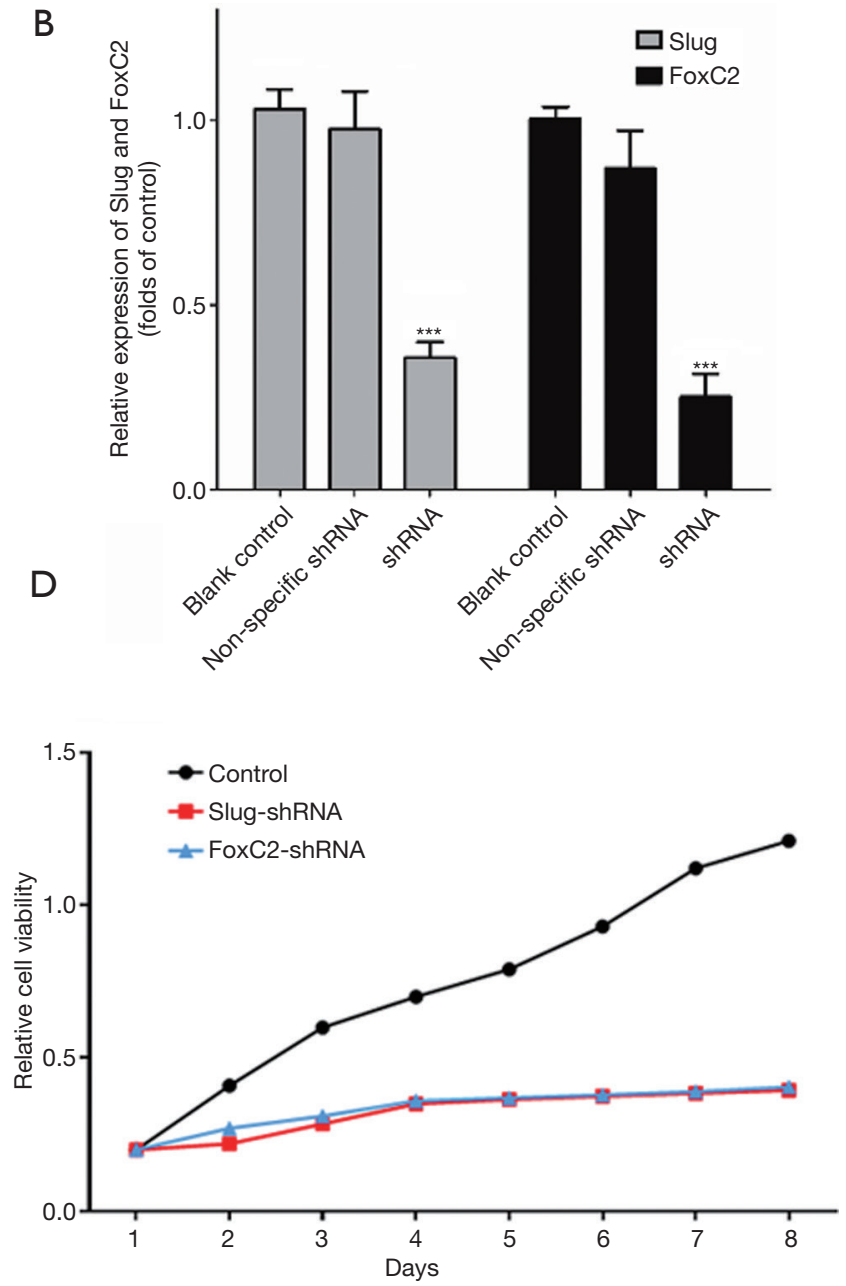

Figure 4 Slug-shRNA and FoxC2-shRNA reduced RL-952cells proliferation. (A) The protein expression in each group was detected using western-blot. Representative images for Slug and FoxC2 were shown; (B) quantification of Slug and FoxC2 expression; (C) the Slug and FoxC2 gene in each group was detected by RT-PCR; (D) RL-952 cell viability was detected on the on the 1-8 day after transfection using CCK-8 method. Data represent the mean $\pm \mathrm{SD}(\mathrm{n}=3)$. ${ }^{* * *}, \mathrm{P}<0.001$ versus control group.

$(\mathrm{P}<0.05)$. This suggests that Slug and FoxC2 may play a crucial role in endometrial carcinoma metastasis and Slug and FoxC2 could be used as an indicator for the prognosis of endometrial carcinoma.

In order to verify the Slug and FoxC2 roles in endometrial carcinoma, Ishikawa and RL-962 endometrial carcinoma cells were transfected with shRNA-Slug and shRNA-FoxC2 to silence the expression of Slug and FoxC2 respectively, which were confirmed by the fluorescence microscopy, Western blotting, and RT-PCR. Cell proliferation, invasion and migration were significantly inhibited by shRNA-Slug and shRNA-FoxC2 in both cell lines. The matrix metalloproteinases (MMP) family is a class of $\mathrm{Zn}^{2+}$ and $\mathrm{Ca}^{2+}$-dependent endopeptidases that play an important role in multiple pathological processes such as inflammation, tumors, and cardiovascular diseases. It has found that MMP-2 and MMP-9 can promote tumor neovascularization in vivo, and upregulation of MMP-2 and MMP-9 in stromal cells could significantly increase the tumor cell metastasis (13). In the current study, our results also highlighted that the expressions of MMP-2 and MMP9 in both cell lines after shRNA-Slug and shRNA-FoxC2 transfection was significantly decreased compared with the control group. 


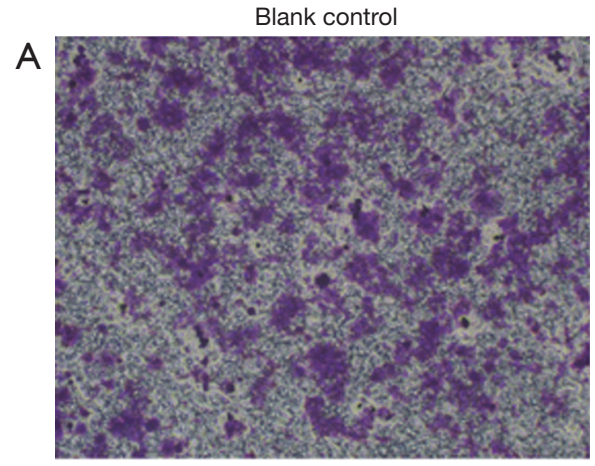

Blank control

B

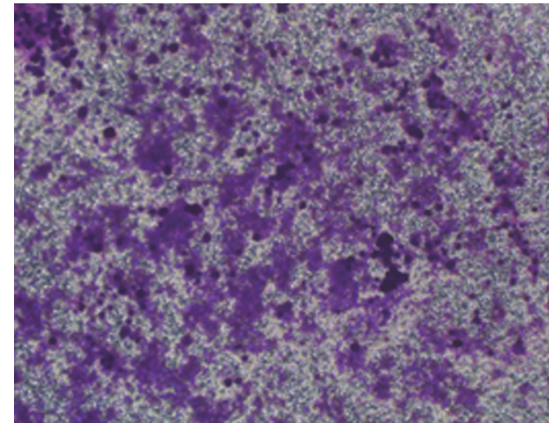

Slug-shRNA

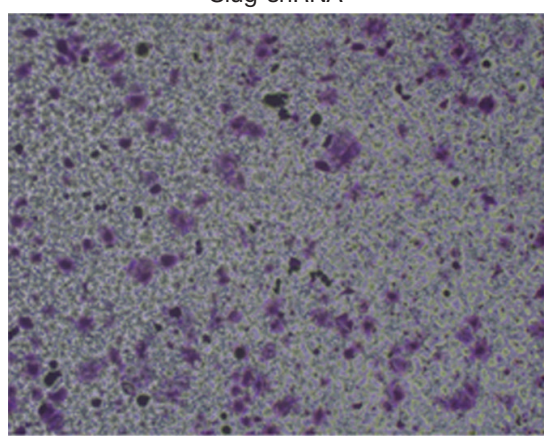

Slug-shRNA

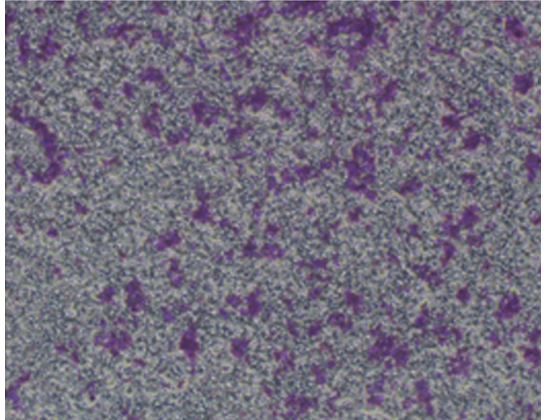

FoxC2-shRNA

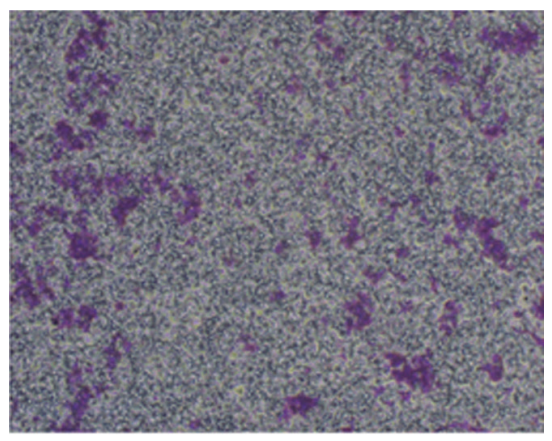

FoxC2-shRNA

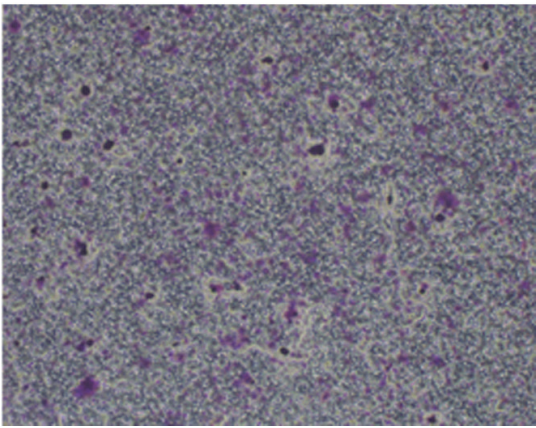

Figure 5 In vitro analysis of migration capacities (A) and invasion capacities (B) of Ishikawa cells after transfected with Slug-shRNA and FoxC2-shRNA $(\times 100)$.

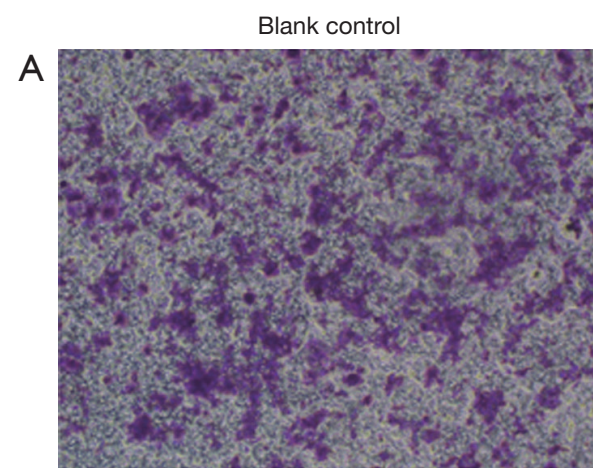

Blank control

B

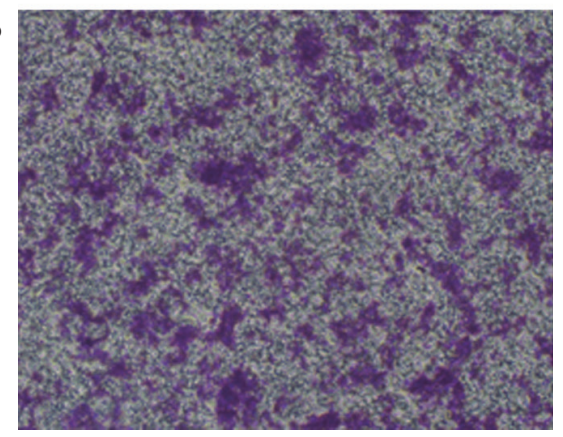

Slug-shRNA

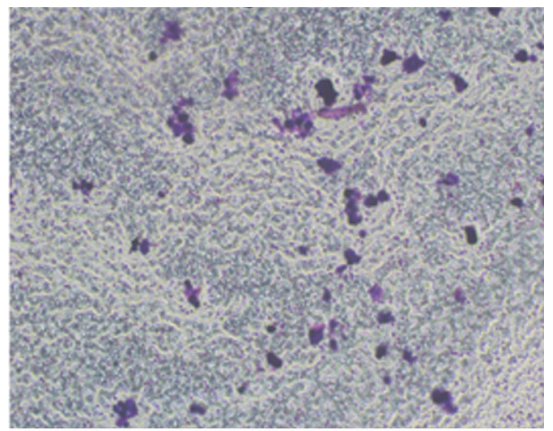

Slug-shRNA

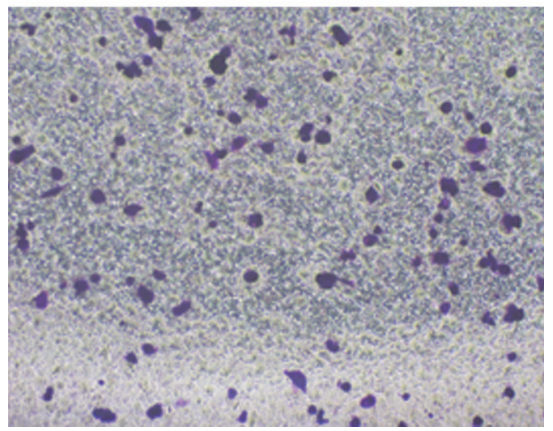

FoxC2-shRNA

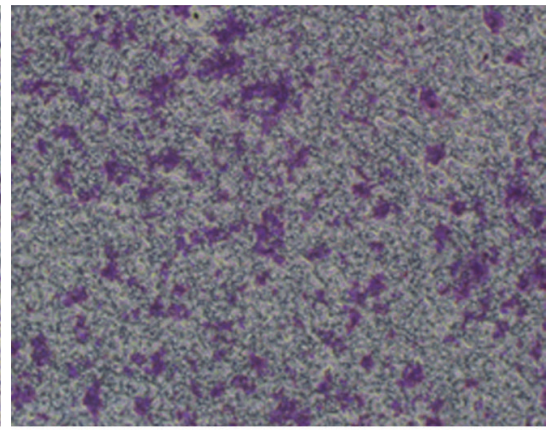

FoxC2-shRNA

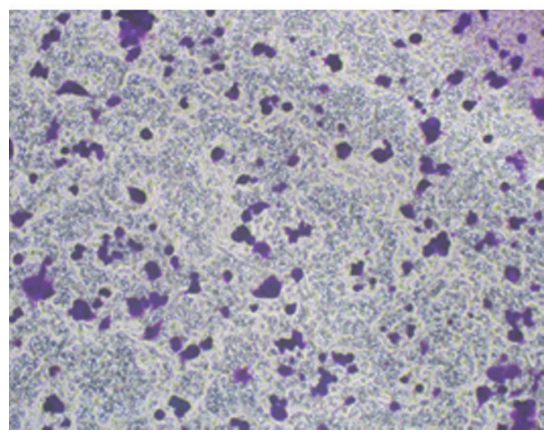

Figure 6 In vitro analysis of migration capacities (A) and invasion capacities (B) of RL-952 cells after transfected with Slug-shRNA and FoxC2-shRNA $(\times 100)$. 

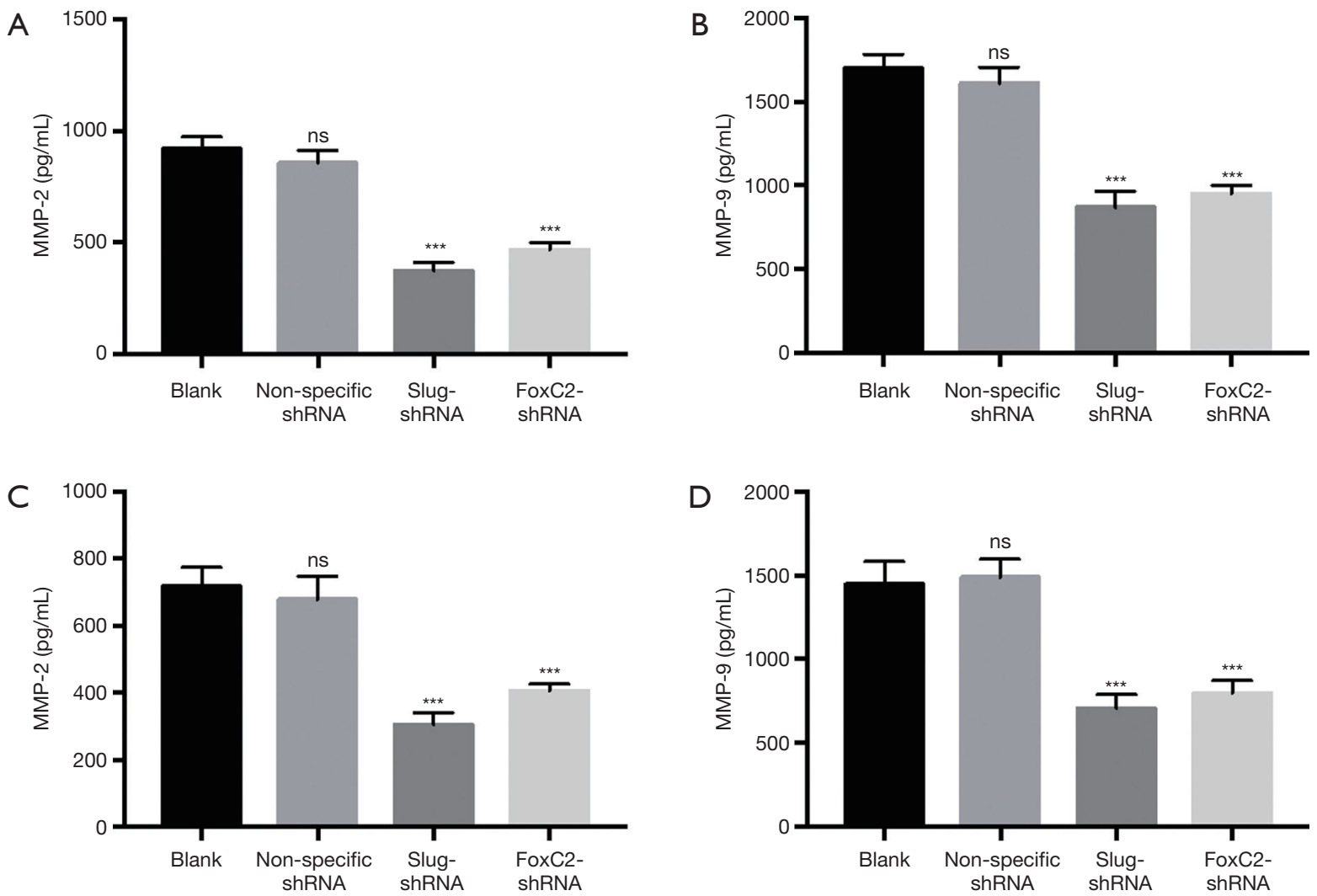

Figure 7 MMP-2 and MMP-9 concentrations in Ishikawa cells (A) and RL-952 cells (B) were detected using Elisa assay. Data represent the mean $\pm \mathrm{SD}(\mathrm{n}=3)$. ${ }^{* *}, \mathrm{P}<0.001$ versus control group; ns, no statistical significance.

Infiltration and metastasis are also the main causes of treatment failure and poor clinical prognosis of endometrial carcinoma. It has been reported that metastasizes of endometrial carcinoma related to by EMT was induced by Slug and FoxC2 (14-19). EMT was an originally physiological process that occurred during the development of mammalian embryos, and recent research showed that EMT played an important role in the metastasis of tumors. Epithelial-derived tumor cells can be transformed into tumor cells with stronger migration ability and the enhanced invasions could also be promoted by interstitial cells. EMT could be activated by both intracellular signaling pathways and integrin signaling pathways, such as Slug, Snail, Twist and FoxC2 (20-25). Then the expressions EMT markers (such as Slug, Snail, and FoxC2) in tumor cells will be significantly increased, which will enhance cell invasiveness and lead to transformation into tumor cells $(11,26,27)$, and the migration and resistance to apoptosis of the tumor epithelial cells will be enhanced (28-30). As EMT markers, E-cadherin, $\mathrm{N}$-cadherin and Vimentin were analyzed in each group in both cell lines. We found that, after transfection of shRNA-Slug and shRNA-FoxC2, the expression of E-cadherin was markedly increased while the N-cadherin and Vimentin expression was significantly decreased, which was in consistence with previous studies $(5,31)$, indicating the suppression of EMT by shRNA-Slug and shRNA-FoxC2.

\section{Conclusions}

In summary, our current study found that, compared with normal endometrial tissues, the Slug and FoxC2 expression levels in endometrial carcinoma tissues were remarkably increased. Interfering with Slug and FoxC2 through shRNAs can effectively suppress the in vitro proliferation, invasion, and migration of Ishikawa and RL-952cells, which may be related to the downregulation of MMPs protein and inhibition of EMT. Further studies will more focus on the detailed mechanism of the regulation of EMT in endometrial carcinoma by Slug and FoxC2. 
A

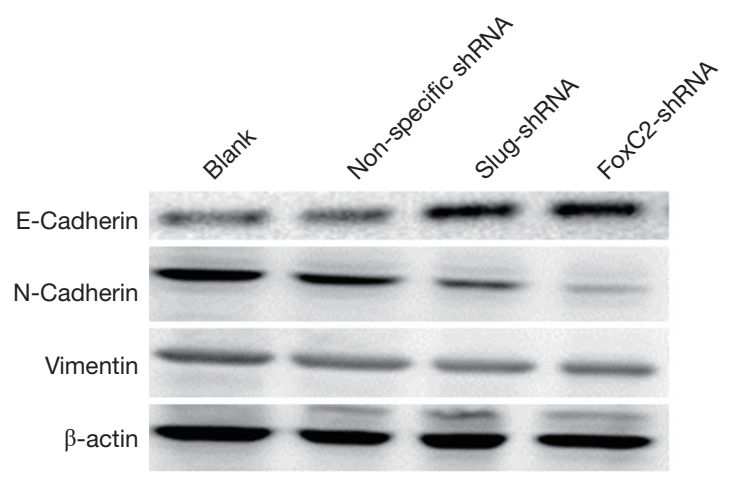

C

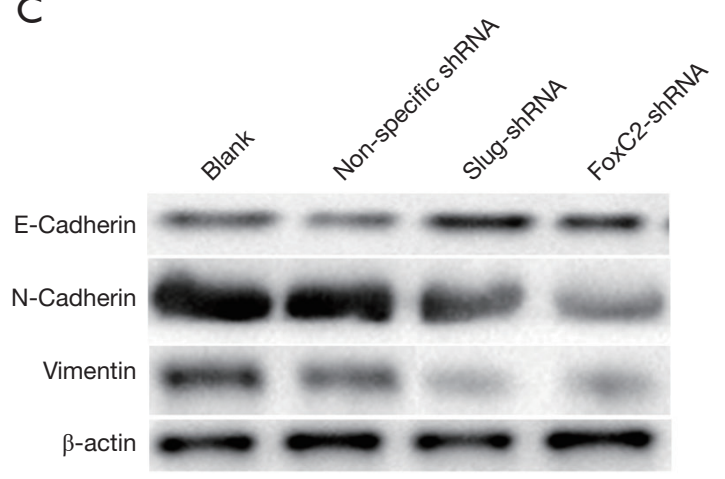

B
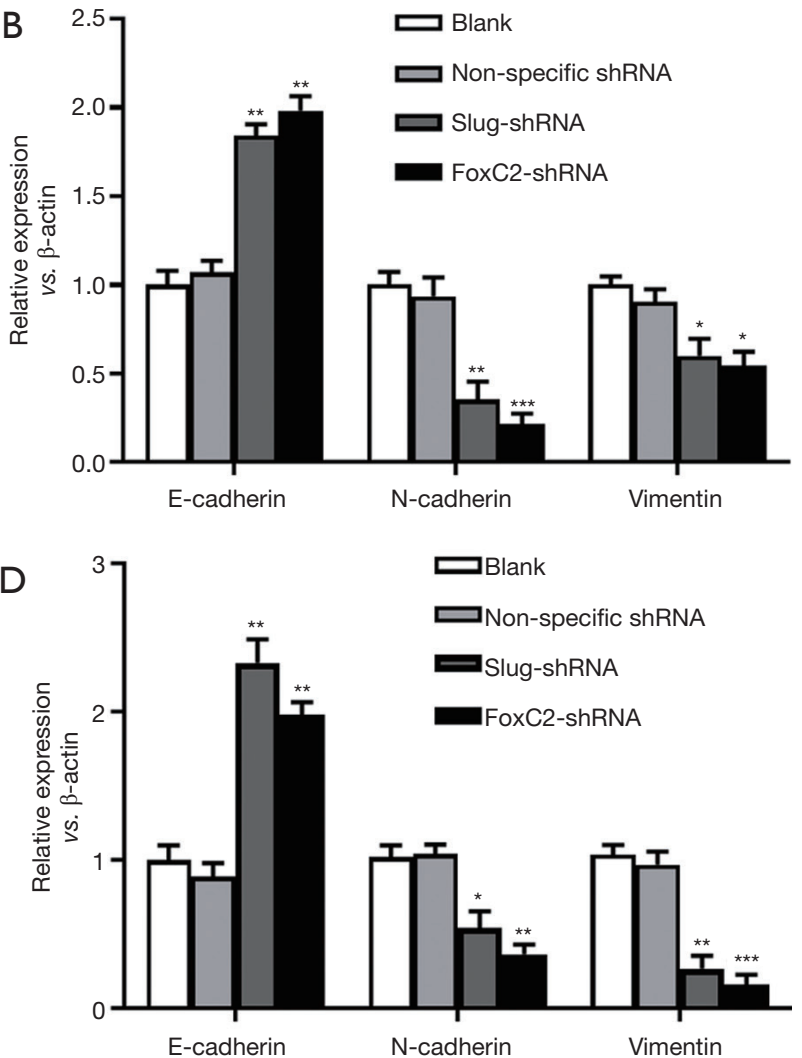

Figure 8 The expression of E-cadherin, N-cadherin and Vimentin in Ishikawa cells (A) and RL-952 cells (B) were detected by Western blotting. The representative images were shown and quantifications were performed. Data represent the mean $\pm \operatorname{SD}(n=3)$. ${ }^{*}<0.05$, ${ }^{* *} \mathrm{P}<0.01,{ }^{* * *} \mathrm{P}<0.001$ versus control group.

\section{Acknowledgments}

Funding: This work was supported by the National Innovation and Entrepreneurship Program for College Students (201810367025) and Natural Science Foundation of Bengbu Medical College (BYKY1822ZD).

\section{Footnote}

Reporting Checklist: The authors have completed the MDAR checklist. Available at http://dx.doi.org/10.21037/tcr-20809

Data Sharing Statement: Available at http://dx.doi. org/10.21037/tcr-20-809

Conflicts of Interest: All authors have completed the ICMJE uniform disclosure form (available at http://dx.doi. org/10.21037/tcr-20-809). The authors have no conflicts of interest to declare.

Ethical Statement: The authors are accountable for all aspects of the work in ensuring that questions related to the accuracy or integrity of any part of the work are appropriately investigated and resolved. The study was conducted in accordance with the Declaration of Helsinki (as revised in 2013). The study was approved by institutional ethics board of The First Affiliated Hospital of Bengbu Medical College (No. BBMEC-2018-10) and informed consent was taken from all the patients.

Open Access Statement: This is an Open Access article distributed in accordance with the Creative Commons Attribution-NonCommercial-NoDerivs 4.0 International License (CC BY-NC-ND 4.0), which permits the noncommercial replication and distribution of the article with the strict proviso that no changes or edits are made and the original work is properly cited (including links to both the 
formal publication through the relevant DOI and the license). See: https://creativecommons.org/licenses/by-nc-nd/4.0/.

\section{References}

1. Phillips S, Kuperwasser C. SLUG: Critical regulator of epithelial cell identity in breast development and cancer. Cell Adh Migr 2014;8:578-87.

2. Kihara A, Wakana K, Kubota T, et al. SLUG expression is an indicator of tumour recurrence in high-grade endometrial carcinomas. Histopathology 2016;69:374-82

3. Zhao Z, Sun YS, Chen W, et al. Hispolon inhibits breast cancer cell migration by reversal of epithelial-tomesenchymal transition via suppressing the ROS/ERK/ Slug/E-cadherin pathway. Oncol Rep 2016;35:896-904.

4. Liu B, Han SM, Tang XY, et al. Overexpressed FoxC2 in ovarian cancer enhances the epithelial-to-mesenchymal transition and invasion of ovarian cancer cells. Oncol Rep 2014;31:2545-54.

5. Du J, Zhang XP, Zhou HJ, et al. Alex3 suppresses nonsmall cell lung cancer invasion via AKT/Slug/E-cadherin pathway. Tumour Biol 2017;39:1010428317701441.

6. Wu DW, Lee MC, Hsu NY, et al. FHIT loss confers cisplatin resistance in lung cancer via the AKT/NF- $\mathrm{kB} /$ Slug-mediated PUMA reduction. Oncogene 2015;34:2505.

7. Anzai E, Hirata K, Shibazaki M, et al. FOXA1 Induces E-Cadherin Expression at the Protein Level via Suppression of Slug in Epithelial Breast Cancer Cells. Biol Pharm Bull 2017;40:1483-89.

8. Uygur B, Abramo K, Leikina E, et al. SLUG is a Direct Transcriptional Repressor of PTEN Tumor Suppressor. Prostate 2015;75:907-16.

9. Sánchez-Duffhues G, de Vinuesa AG, Lindeman JH, et al. SLUG Is Expressed in Endothelial Cells Lacking Primary Cilia to Promote Cellular Calcification. Arterioscler Thromb Vasc Biol 2015;35:616-27.

10. Jiang F, Zhou L, Wei C, et al. Slug inhibition increases radiosensitivity of oral squamous cell carcinoma cells by upregulating PUMA. Int J Oncol 2016;49:709-19.

11. Zhang CL, Zhu KP, Ma XL. Antisense lncRNA FoxC2AS1 promotes doxorubicin resistance in osteosarcoma by increasing the expression of FoxC2. Cancer Lett 2017;396:66-75.

12. Paranjape AN, Soundararajan R, Werden SJ, et al. Inhibition of FoxC2 restores epithelial phenotype and drug sensitivity in prostate cancer cells with stem-cell properties. Oncogene 2016;35:5963-76.

13. Cui YM, Jiao HL, Ye YP, et al. FoxC2 promotes colorectal cancer metastasis by directly targeting MET. Oncogene 2015;34:4379-90.

14. Jiang W, Fan H, Qian C, et al. Prognostic value of high FoxC2 expression in resectable non-small cell lung cancer, alone or in combination with E-cadherin expression. BMC Cancer 2016;16:16.

15. Wu B, Wei J, Hu Z, et al. Slug silencing inhibited perineural invasion through regulation of EMMPRIN expression in human salivary adenoid cystic carcinoma. Tumour Biol 2016;37:2161-69.

16. Zheng M, Jiang YP, Chen W, et al. Snail and Slug collaborate on EMT and tumor metastasis through miR101-mediated EZH2 axis in oral tongue squamous cell carcinoma. Oncotarget 2015;6:6797-810.

17. Cappellesso R, Marioni G, Crescenzi M, et al. The prognostic role of the epithelial-mesenchymal transition markers E-cadherin and Slug in laryngeal squamous cell carcinoma. Histopathology 2015;67:491-500.

18. Tsukasa K, Ding Q, Yoshimitsu M, et al. Slug contributes to gemcitabine resistance through epithelial-mesenchymal transition in CD133(+) pancreatic cancer cells. Hum Cell 2015;28:167-74.

19. Cai J, Tian AX, Wang QS, et al. FOXF2 suppresses the FoxC2-mediated epithelial mesenchymal transition and multidrug resistance of basal-like breast cancer. Cancer Lett 2015;367:129-37.

20. Zhou Z, Zhang L, Xie B, et al. FoxC2 promotes chemoresistance in nasopharyngeal carcinomas via induction of epithelial mesenchymal transition. Cancer Lett 2015;363:137-45.

21. Liu B, Han SM, Tang XY, et al. Overexpressed FoxC2 in ovarian cancer enhances the epithelial-to-mesenchymal transition and invasion of ovarian cancer cells. Oncol Rep 2014;31:2545-54.

22. Merikallio H, Turpeenniemi-Hujanen TT, Paakko P, et al. Slug is associated with poor survival in squamous cell carcinoma of the lung. Int J Clin Exp Pathol 2014;7:5846-54.

23. Zhang T, Liang L, Liu X, et al. TGF beta 1-Smad3Jagged1-Notch1-Slug signaling pathway takes part in tumorigenesis and progress of tongue squamous cell carcinoma. J Oral Pathol Med 2016;45:486-93.

24. Sun TY, Xie HJ, Li Z, et al. Expression of FoxC2 in renal cell carcinoma and its relationship to clinical pathological features. Int J Clin Exp Med 2015;8:13388-92.

25. Li C, Ding H, Tian J, et al. Forkhead Box Protein C2 (FoxC2) Promotes the Resistance of Human Ovarian Cancer Cells to Cisplatin In Vitro and In Vivo. Cell 
Physiol Biochem 2016;39:242-52.

26. Xue M, Zhu FY, Chen L, et al. HoxB9 promotes the migration and invasion via TGF-beta1/Smad2/Slug signaling pathway in oral squamous cell carcinoma. Am J Transl Res 2017;9:1151-61.

27. Yang J, Ren YX, Shang Y'T, et al. Effect of Slug and Sox9 on the Invasion and Metastasis of Nasopharyngeal Carcinoma Stem Cells. J Kunming Med Univ 2016;37:52-55.

28. Yang C, Cui XX, Dai XQ, et al. Downregulation of FoxC2 enhances apoptosis induced by 5 -fluorouracil through activation of MAPK and AKT pathways in colorectal cancer. Oncol Lett 2016;11:1549-54.

Cite this article as: Zhu Q, Tang M, Wu L. Expression of combined interference of slug and FoxC2 in endometrial carcinoma and its clinicopathological relationship. Transl Cancer Res 2020;9(9):5268-5280. doi: 10.21037/tcr-20-809
29. Xu T, Fan B, Lv CJ, et al. Slug mediates nasopharyngeal carcinoma radio résistance via downregulation of PUMA in a 553 -dependent and -independent manner. Oncol Rep 2015;33:2631-38.

30. Jittreetat T, Shin YS, Hwang HS, et al. Tolfenamic Acid Inhibits the Proliferation, Migration, and Invasion of Nasopharyngeal Carcinoma: Involvement of p38-Mediated Down-Regulation of Slug. Yonsei Med J 2016;57:588-98.

31. Kanady JD, Munger SJ, Witte MH, et al. Combining FoxC2 and Connexin 37 deletions in mice leads to severe defects in lymphatic vascular growth and remodeling. Dev Biol 2015;405:33-46. 